Clío América/ Vol. 13, No. 25 - 2019 / 255 - 263

DOI: http://dx.doi.org/10.21676/23897848.3266

\title{
ESTRÉS POSTRAUMÁTICO Y APTITUDES DE EMPRENDIMIENTO Y RESILIENCIA EN MUJERES VÍCTIMAS DEL CONFLICTO ARMADO
}

\section{POST-TRAUMATIC STRESS AND APTITUDES OF ENTREPRENEURSHIP AND RESILIENCE IN VICTIM WOMEN OF THE ARMED CONFLICT}

\author{
Kethy Luz Pérez-Correa ${ }^{1(\mathbb{D})}$, Oscar Eduardo Rodríguez-Vega ${ }^{2}$ y Belia Elena Linero-Gómez ${ }^{3}$ (i) \\ ${ }^{1}$ Universidad Cooperativa de Colombia - Sede Santa Marta.Email:k1_perez@hotmail.com \\ ${ }^{2}$ Universidad Cooperativa de Colombia - Sede Santa Marta. Email: oscar.rodriguez@ucc.edu.co \\ ${ }^{3}$ Universidad Cooperativa de Colombia - Sede Santa Marta. Email: belia.linero@ campusucc.edu.co
}

Para citar este artículo: (2019). Relación del estrés postraumático con las aptitudes de emprendimiento y resiliencia en mujeres víctimas del conflicto armado. Clío América, 13(25), 255-263. doi: http://dx.doi.org/10.21676/23897848.3266

Recibido: 22 febrero de 2019

Aceptado: 17 de mayo de 2019

Publicado en línea: mayo 24 de 2019

Palabras clave:

estrés

postraumático;

emprendimiento;

resiliencia;

víctimas;

conflicto armado.

JEL: M10.

\section{RESUMEN}

El presente artículo tiene por objeto explorar la relación existente entre el estrés postraumático y las aptitudes de emprendimiento y resiliencia en mujeres víctimas del conflicto armado en cinco municipios del departamento de Magdalena, Colombia. El diseño de la investigación es no experimental, transversal y de campo. La población objeto de estudio fue de 241 mujeres víctimas del conflicto armado, con emprendimientos en desarrollo empresarial y social. En los resultados se observó que la resiliencia en las mujeres emprendedoras del Magdalena se asume como la capacidad de sobreponerse y enfrentar las situaciones adversas. Por otro lado, se encontró que mediante el emprendimiento se promueve la competitividad a nivel personal, familiar y social teniendo en cuenta estrategias de colectividad y trabajo en equipo de la comunidad. Sin embargo, persisten muchas problemáticas para lograr la efectividad deseada. En términos de conclusiones, se hace énfasis en la necesidad de articular esfuerzos de diversos sectores que contribuyan a mejorar las condiciones de las mujeres víctimas del conflicto armado.

\section{ABSTRACT}

The purpose of this research is to explore the relationship between post-traumatic stress and entrepreneurial skills and resilience in women victims of armed conflict in 5 municipalities of the department of Magdalena, Colombia. The design of the research is non-experimental, cross-sectional and field-based. The population under study was 241 women victims of the armed conflict and with undertakings in business and social development. In the results was observed that the resilience in the enterprising women of the Magdalena is assumed like the capacity to overcome and to face the adverse situations. On the other hand, it was found that entrepreneurship promotes personal, family and social competitiveness, taking into account collective strategies and teamwork in the community. However, many problems persist to achieve the desired effectiveness. In terms of conclusions, emphasis is placed on the need to coordinate the efforts of various sectors that contribute to improve the conditions of women victims of the armed conflict. 


\section{ESTRÉS POSTRAUMÁTICO Y APTITUDES DE EMPRENDIMIENTO Y RESILIENCIA EN MUJERES VÍCTIMAS DEL CONFLICTO ARMADO}

\section{INTRODUCCIÓN}

La historia de Colombia ha sido marcada por las implicaciones sociales, económicas y políticas que ha tenido el conflicto armado en los últimos años (Arias, Camacho, Ibáñez, Mejía y Rodríguez, 2014). Sierra (2019) expresa que, aunque formalmente el conflicto armado tiene sus inicios en los sesenta del siglo XX, sus orígenes se encuentran en 1948, cuando inicia la guerra civil partidista que genera las dos grandes guerrillas colombianas: las Fuerzas Armadas Revolucionarias de Colombia - FARC y el Ejército de Liberación Nacional - ELN. Sin embargo, Yaffe (2011) sostiene que desde ese período han sido múltiples los sectores que han participado en el conflicto armado colombiano: "las guerrillas de izquierda, los paramilitares de derecha, los narcotraficantes, el gobierno, las fuerzas armadas y la sociedad Civil” (p. 191).

Según Valencia y Daza (2010), todos los colombianos han sido víctimas del conflicto de manera directa o indirecta y han sido marcados por consecuencias físicas o psicológicas. Sin embargo, de las víctimas directas del conflicto, un porcentaje significativo corresponde a personas del sexo femenino. En datos de la Unidad de Víctimas se expresa por Jara (2017) que las mujeres representan el 49,73\% de las 8347566 víctimas, es decir 4151416 . Adicionalmente, cuando se explora la población de mayor edad se encuentra que por cada 96 hombres víctimas del conflicto existen 100 mujeres afectadas (Correa et al., 2015). En este sentido, Tabares (2011) expone que los acontecimientos violentos modifican los sentidos y prácticas de las personas al producir alteraciones en sus discursos y las formas de relacionarse en la sociedad. Adicionalmente, Castañeda y Camargo (2018) demostraron que el conflicto armado tiene repercusiones en la salud mental de las personas, además de las reiteradas violaciones a los derechos humanos (Lubell, 2005; Ramírez, 2016; Singh, 2019).

Una de estas afectaciones en la salud mental de las personas se genera por el estrés postraumático que se desprende de las acciones y experiencias del conflicto, que se puede manifestar hasta 10 años después de los procesos conflictivos y con mayor intensidad en los contextos geográficos que tuvieron mayor exposición al conflicto armado (Gómez et al., 2016). Aunque son pocos los estudios que se han realizado en Colombia para determinar el nivel de esta patología en víctimas de dicho conflicto, y mucho menos se ha ejecutado una investigación con representatividad a nivel nacional (Escobar, Folino y
Schmalbach, 2006), según Sinisterra, Figueroa, Moreno, Robayo y Sanguino (2010) solo el 35,50 \% de los hombres víctimas en Bogotá presenta una prevalencia del trastorno de estrés postraumático, mientras que en las mujeres esta cifra corresponde al 64,50\%. Así mismo, Cudris y Barrios (2018) en su investigación han encontrado que las mujeres veteranas en la guerra reportaron dos de tres síntomas asociados con el estrés postraumático.

Es evidente que existen unas consecuencias asociadas a las dinámicas de las confrontaciones armadas en la población colombiana y de manera especial en las mujeres. Esto ha generado unas reacciones por parte de las personas para responder a las necesidades propias de la violencia, como los emprendimientos y las agrupaciones sociales (Riaño, 2006; Reyes y Fattori, 2019). Para Guevara y Barney (2009), la necesidad de generar iniciativas económicas por parte de las mujeres corresponde a dos factores: el primero se relaciona con el tránsito del mundo rural al urbano que pone en evidencia unas capacidades laborales de las mujeres, invisibilizadas por las tareas del campo, y el segundo corresponde a la necesidad de generar ingresos para garantizar la existencia familiar.

En la investigación desarrollada por Correa et al. (2015) se manifiesta que mientras un $67 \%$ de los hombres lograba ocuparse laboralmente, solo el $19 \%$ conseguía este meta. Por su parte, Riaño (2006) encontró que las mujeres pertenecientes a núcleos familiares víctimas del conflicto armado se dedican fundamentalmente a las labores domésticas de madre y esposa, centrando su atención en el cuidado de los individuos que conforman la familia. Mientras tanto, Botello (2000) sostiene que las mujeres manifiestan sentimientos de frustración por no poder aportar, en términos económicos, a resolver las necesidades del hogar. Por su parte, Andrade y Sicachá (2012) exponen que, como consecuencia de diversos factores asociados al conflicto, especialmente la desaparición de los hombres en acciones de guerra y la necesidad de incrementar los ingresos familiares por parte de las familias afectadas, se han invertido los roles tradicionales de la mujer como cuidadora para posicionarla como sujeto de aportaciones económicas, promoviendo unos roles y actitudes como el emprendimiento. En esta misma línea se expresa que las mujeres tienen mayor sensibilidad ante los sufrimientos generados por el conflicto a las víctimas, por lo tanto, consideran que "la única vía para emprender luchas comunes y lograr generar cambios es bajo el principio de la asociatividad, por lo que deciden organizarse bajo los lazos de cooperación y colaboración" (Barros y Rojas, 
2015, p. 14).

Para Viloria, Daza y Miranda (2016), el emprendimiento corresponde a los comportamientos de las personas que les permiten tomar decisiones y generar innovaciones en los contextos organizacionales, es decir que se encuentra relacionado con los patrones psicológicos de las personas y, por lo tanto, estará también determinado por los niveles de salud mental de estas. Según Cortez (2018), se podría planear que las víctimas asumen el emprendimiento como una alternativa para mejorar la calidad de vida, evidenciándose así una actitud resiliente en las personas que han sufrido las consecuencias del conflicto armado. Es otras palabras, el emprendimiento se configura como una oportunidad para superar las adversidades. En este sentido, diversos estudios han demostrado que existe una relación entre la resiliencia y el emprendimiento (Hmieleski y Carr, 2008; Williams, Vorley y Ketikidis, 2013; Romero, Jaramillo, Aguirre y Ruiz, 2017).

A partir de lo anterior, esta investigación se torna relevante en la medida en que hace una lectura explicativa de las características de resiliencia y emprendimiento en mujeres víctimas del conflicto armado, permitiendo acercarse a la realidad social y económica de las mismas y generar puntos de partida para tomar decisiones en perspectiva de mejorar sus condiciones de vida. Desde allí se espera generar insumos para que tanto el Estado como la sociedad civil y el sector empresarial aporten a generar condiciones efectivas para el éxito de las acciones emprendedoras de las víctimas del conflicto, ya que este no depende solo de las capacidades y habilidades personales, sino que también se influencia de las condiciones y ecosistemas para el emprendimiento (Uribe, 2017). Así mismo, es claro que la generación de empresas se convierte en una posibilidad para reducir la pobreza de las personas (Rodríguez y Salazar, 2015; Álvarez, González y Vergara, 2016).

\section{METODOLOGÍA}

El diseño de esta investigación fue no experimental, transversal y de campo. Esta siguió un ciclo desde lo exploratorio hasta lo explicativo que buscó el porqué de los hechos a través de relaciones causa-efecto. El paradigma de la investigación fue cuantitativo y tuvo un enfoque empírico inductivo.

La población objeto de estudio fue de 241 mujeres víctimas del conflicto armado y con emprendimientos en desarrollo empresarial y social, ubicadas geográficamente en los municipios de Salamina, El Banco, Fundación, Plato y Santa Marta dentro del departamento del Magdalena, Colombia.

Se verificó en esta población el cumplimiento de TEPT (Trastorno de Estrés Postraumático) mediante la Lista de Chequeo para TEPT (Weathers, Litz, Herman, Huska y Keane, 1993). Para explorar los acontecimientos que se asocian al trauma fue utilizada la Lista de Chequeo de Eventos (Blake, et al., 1990). Por su parte, para la identificación de la relación entre el TEPT con las aptitudes de emprendimiento y resiliencia se diseñó un instrumento con respuesta escala tipo Likert. Para medir la validez y la confiabilidad de este se utilizó el Coeficiente de Alpha Cronbach $\mathrm{rtt}=0,98 \mathrm{y}$ el juicio de 10 expertos.

Inicialmente, se desarrolló un proceso de revisión y fundamentación teórica de cada una de las variables de la investigación, buscando identificar las tendencias actuales de la problemática en el contexto nacional y mundial. Así mismo, se definieron los instrumentos de recolección de información. Seguidamente, se seleccionaron las personas que harían parte del estudio y se aplicaron los instrumentos.

La información recolectada fue sistematizada en matrices y se procesó con el apoyo de la estadística inferencial. El software utilizado correspondió al estadístico SPSS.

La investigación se desarrolló aplicando las normas de la Declaración de Helsinki de 1975, Convenio 169 de la OIT (Organización Internacional del Trabajo) y la Resolución 8430 de 1993 del Ministerio de Salud de Colombia.

\section{RESULTADOS}

En términos de los resultados encontrados en la investigación, los datos de la evaluación de la resiliencia se muestran en la Tabla 1.

Tabla 1. Resiliencia en mujeres víctimas del conflicto armado

\begin{tabular}{|c|l|r|r|r|r|}
\hline Variable Resiliencia & & \multicolumn{1}{|c|}{ Media } & \multicolumn{1}{c|}{ Desviación típ. } & Error típ. de la media \\
\hline Puntaje & Modalidades & 5 & 1,93 & 0,20857 & 0,09327 \\
\cline { 2 - 6 } & Beneficios & 4 & 1,945 & 0,1561 & 0,07805 \\
\hline
\end{tabular}

Fuente: elaboración propia.

La resiliencia, entendida como un factor protector, se observó en las mujeres emprendedoras del Magdalena 


\section{ESTRÉS POSTRAUMÁTICO Y APTITUDES DE EMPRENDIMIENTO Y RESILIENCIA EN MUJERES VÍCTIMAS DEL CONFLICTO ARMADO}

como la capacidad de sobreponerse y enfrentar las situaciones adversas, generando con ello una nueva perspectiva socio-cultural que determina las estrategias de afrontamiento y las acciones de estas ante el manejo del estrés y la dinámica del conflicto. Esto incide en el planteamiento de nuevas dinámicas de subsistencias sociales, políticas y económicas, donde el emprendimiento y la motivación intrínseca por superar y sobreponerse al daño generan cambios a nivel personal. Entre dichos cambios se encuentran el autocontrol, la regulación emocional, la capacidad de adaptación y el amor por los suyos.

Por su parte, la evaluación de la variable de emprendimiento se detalla en la Tabla 2.

Tabla 2. Emprendimiento en mujeres víctimas del conflicto armado.

\begin{tabular}{|l|l|l|l|l|l|}
\hline Variable de Emprendimiento & N & Media & Desviación típ. & Error típ. de la media \\
\hline Puntaje & Estrategas Personales & 3 & 2,0967 & 0,09292 & 0,05364 \\
\cline { 2 - 6 } & $\begin{array}{l}\text { Fuerzas que Moldean la } \\
\text { Resiliencia }\end{array}$ & 4 & 2,008 & 0,08349 & 0,03734 \\
\hline
\end{tabular}

Fuente: elaboración propia.

Se evidencia que a través del emprendimiento se pueden lograr niveles de competitividad a nivel personal, familiar y social de las participantes, teniendo en cuenta estrategias de colectividad y trabajo en equipo de la comunidad. Es importante reconocer que la creación de ventanas de emprendimientos sociales estimula el desarrollo personal de las mujeres víctimas del conflicto armado y desarrollan la capacidad del autosostenimiento en cuanto a la relación de trabajo mancomunado.

El resumen del modelo empleado se detalla en la Tabla 3.

Tabla 3. Resumen del modelo.

\begin{tabular}{|l|l|l|l|l|}
\hline Modelo & $\mathbf{R}$ & R cuadrado & R cuadrado corregida & Error típ. de la estimación \\
\hline $\mathbf{1}$ & $0,687 \mathrm{a}$ & 0,472 & 0,460 & 8,965 \\
\hline
\end{tabular}

Fuente: elaboración propia.

Sin embargo, los emprendimientos con alto grado de desarrollo se encuentran de manera poco suficiente, lo que implica que casi nunca se establecen planes de negocio organizados. Pese a ello, existe un alto grado de emprendimientos de subsistencia en la región que obedecen a la variable resiliencia. En cuanto a la aplicación del TEP, se pude revisar que el $67 \%$ de las mujeres sujeto de estudio cumple con criterios para la clínica del mismo, en relación al $100 \%$ de la población estudiada. Se pudo observar entonces que, a pesar del estrés postrauma de la mujeres víctimas del conflicto armado, ellas poseen altas aptitudes para la subsistencia, por lo que han desarrollado emprendimientos con poca escala de desarrollo. Esto está asociado al bajo nivel de escolaridad, la escasa asistencia psicosocial y el poco reconocimiento desde su dinámica funcional como individuo en el contexto social.

Las condiciones revisadas en el estudio y las de la baja escalabilidad de los emprendimientos encontrados que permea a la población, tienen características psicológicas de subsistencia, lo que lleva a la generación de cooperativas y pequeños emprendimientos. Es de resaltar que el $67 \%$ de mujeres con claros rasgos de estrés postraumático representa a mujeres desarrolladoras de emprendimientos.
Esto genera un cruce de variables interdependientes donde la capacidad de resiliencia es alta frente al postrauma.

\section{DISCUSIÓN}

El conflicto armado en Colombia ha marcado la historia del país y ha generado innumerables consecuencias negativas en la vida de las personas, especialmente en quienes se han encontrado en zonas de alta vulnerabilidad social (Pérez y Salas, 2019). Esto ha planteado la necesidad en las víctimas de buscar alternativas que les permitan sobreponerse a las situaciones adversas y encontrar modos para mejorar sus condiciones de vida. En este sentido, emerge el emprendimiento como una posibilidad para generar ingresos en las familias por parte de las mujeres que han vivido las dinámicas agresivas del conflicto.

Esta investigación se desarrolló con el objetivo de explorar los niveles de resiliencia y emprendimiento en mujeres víctimas del conflicto armado en cinco municipios del departamento del Magdalena, que corresponden a Salamina, El Banco, Fundación, Plato y Santa Marta. Así mismo, buscó determinar la relación entre tales variables, 
ya que, según Cadavid (2014), las mujeres han sido un blanco principal del conflicto en Colombia.

Se encontró en esta investigación que la resiliencia en las mujeres víctimas del conflicto armado se convierte en una herramienta para enfrentar las situaciones difíciles que se han vivido. Dentro de las estrategias que utilizan las mujeres para superarlas se encuentran el autocontrol, la regulación emocional, la capacidad de adaptación y el amor por los suyos. Una investigación que se había realizado con mujeres residentes en Bogotá identificó que usaban estrategias similares como el autoconocimiento y la autogestión en el restablecimiento de sus proyectos de vida, y la espiritualidad como claves para enfrentar el estrés postraumático generado por el conflicto (Albarracín y Contreras, 2017). Por otro lado, Castillo y Palma (2016) encontraron en su investigación en mujeres viudas y víctimas del conflicto armado, que acuden a la configuración de valores como la solidaridad, la cooperación, la reciprocidad y la confianza como estrategias resilientes para sobreponerse a las dificultades. Sin embargo, se ha demostrado que no todas las mujeres logran el mismo nivel de resiliencia. En el estudio de Utria, Amar, Martínez, Colmenares y Crespo (2015) se identificaron tres grupos: las mujeres más resilientes, las mujeres con características limitadas de resiliencia y las mujeres con pocas aptitudes resilientes. Esto implica un reto para los responsables de las políticas públicas y la sociedad, en perspectiva de configurar escenarios que respondan a las necesidades de estos grupos poblacionales.

Adicionalmente, en esta investigación se encontró que las mujeres acuden a los emprendimientos basados en asociatividad y el trabajo colaborativo. En este sentido, Barrera (2018) propone en su investigación que se deben generar los ambientes y condiciones desde el Estado para garantizar la efectividad de la creación de unidades productivas, y que se deben articular los emprendimientos de las víctimas del conflicto a las políticas de desarrollo de la nación. En coherencia con lo anterior, el estudio de Cortez (2018) concluye que los emprendimientos de las mujeres víctimas del conflicto armado se desarrollan de manera informal y aislada, es decir, sin articularse con las dinámicas económicas de las regiones donde se asientan. Por lo tanto, como lo proponía Muñoz (2018), es necesario que estos emprendimientos se fundamenten en las bases del trabajo colaborativo y el consumo cooperativo.

Cuando se explora el emprendimiento social en las mujeres que han sido afectadas por el conflicto, se encuentra que este estimula el desarrollo personal y genera posibilidades para el autosostenimiento a partir de la generación de ingresos. Santamaría (2016) expresó que las actividades económicas funcionan como una terapia ocupacional para las víctimas del conflicto, ya que les permite superarse, educarse y aportar a la generación de posibilidades laborales. También Mariño y Valencia (2015) habían considerado que a partir de estrategias integrales para fomentar el emprendimiento se puede aportar no solo al mejoramiento de las condiciones económicas de las víctimas, sino también a sus dinámicas políticas, sociales y culturales.

El nivel de desarrollo o éxito de los emprendimientos de las víctimas del conflicto se determina en gran medida por el grado de acompañamiento o asistencia psicosocial, el reconocimiento social como actor económico y los niveles de educación y formación de las emprendedoras. Sobre esta última categoría relacionada con los procesos educativos, Romero y Contreras (2015) habían manifestado que el bajo nivel de escolaridad en mujeres pobres víctimas del conflicto aumenta sus posibilidades de permanecer en la pobreza. Por su parte, Moreno y Díaz (2016) alertaban sobre la necesidad de que los procesos de atención psicosocial se enfoquen en el reconocimiento y la dignificación de las personas afectadas por el conflicto. En esto también coinciden las investigaciones de Villa (2012, 2016), Villa, Barrera, Arroyave y Montoya (2017) y Villa, Londoño, Gallego, Arango y Rosso (2016). Para lograr mayores resultados en este punto, Tol et al. (2013) sostienen que deben generarse mayores investigaciones sobre los efectos de las intervenciones.

\section{CONCLUSIÓN}

Evidentemente el conflicto armado en Colombia ha generado afectaciones tanto materiales como psicológicas en la vida de un número significativo de población nacional. Muchas personas han sido desplazadas, asesinadas, desterradas, violadas, amenazadas, entre otras acciones que vulneran sus derechos fundamentales. Una proporción muy significativa de la población formalmente reconocida como víctima corresponde a mujeres, lo que ha generado cambios y transformaciones en sus formas de pensar, relacionarse y sobrevivir. Sin embargo, ha sido un nicho poblacional caracterizado por la capacidad de enfrentarse y encontrar soluciones ante las situaciones adversas. Esta actitud resiliente les ha permitido agruparse y movilizarse en torno a la consecuencia de formas que les permitan generar recursos para mejorar su calidad de vida y la de sus familias. Es así como a través del trabajo colaborativo y la asociación configuran estrategias de emprendimiento.

El emprendimiento ha sido asumido no solo como una posibilidad de generación de ingresos, sino como una 


\section{ESTRÉS POSTRAUMÁTICO Y APTITUDES DE EMPRENDIMIENTO Y RESILIENCIA EN MUJERES VÍCTIMAS DEL CONFLICTO ARMADO}

actitud resiliente que les permite a las mujeres el crecimiento personal y el reconocimiento como actores claves en la sociedad. Esto permite demostrar la relación entre resiliencia y emprendimiento para romper con los efectos traumáticos de la guerra.

El éxito de las actividades e iniciativas de emprendimiento que lideran las mujeres víctimas del conflicto armado depende de diversos factores de acompañamiento estatal y social. Por lo tanto, se hace necesario desarrollar políticas públicas que promuevan la articulación de esfuerzos institucionales en perspectiva de la consolidación de ambientes pertinentes para el emprendimiento y la alineación de las iniciativas productivas con las agendas y las necesidades económicas, políticas, sociales y ambientales de los territorios. Esto permitirá la generación de recursos adecuados para fomentar el desarrollo y el mejoramiento de la calidad de vida las mujeres víctimas del conflicto.

Adicionalmente, se deben fortalecer las estrategias de acompañamiento psicosocial de las víctimas, buscando brindar herramientas claves para la dignificación y la superación de los eventos traumáticos, y generar así las reconstrucciones de los tejidos sociales y el establecimiento de ambientes de paz. Por eso, se propone desarrollar procesos investigativos que evalúen la efectividad de las iniciativas de emprendimiento de las mujeres víctimas del conflicto $\mathrm{y}$, desde allí, generar propuestas de mejoramiento de las estrategias de acompañamiento por parte del Estado y demás actores sociales.

\section{Declaración sobre conflictos de interés}

Los autores declaran que no existe conflicto de intereses en la investigación. Así mismo, que los resultados y afirmaciones académicas expuestas se desprenden del proceso investigativo y no han sido influenciadas por ninguna persona o institución.

\section{REFERENCIAS BIBLIOGRÁFICAS}

Albarracín, L.A. y Contreras, K. (2017). La fuerza de las mujeres: un estudio de las estrategias de resiliencia y la transformación en la ocupación humana de mujeres víctimas del conflicto armado en Colombia. Revista Ocupación Humana, 17(1), 25-38.

Álvarez, S. A., González, D. J. y Vergara, G. L. (2016). El emprendimiento en la Fundación Universitaria Tecnológico Comfenalco 2006-
2015, Clío América, 10(20), 126 - 138. doi: https://doi.org/10.21676/23897848.1872

Andrade, J. A. y Sicachá, M. A. (2012). Indicators of individual and community mental health in women-headed households in situations of forced displacement in the state of Quindío. Revista el Ágora, 12(1), 61-75. doi: https://doi.org/10.21500/16578031.222

Arias, M. A., Camacho, A., Ibáñez, A. M., Mejía, D. y Rodríguez, C. (2014). Costos económicos y sociales del conflicto en Colombia: ¿cómo construir un posconflicto sostenible? Bogotá, Colombia: Ediciones Uniandes-Universidad de los Andes.

Barrera, M. E. (2018). Análisis de las experiencias de intervención para creación de unidades productivas a jóvenes entre 15 a 24 años en Colombia entre 2010-2016 (tesis de maestría). Universidad Militar Nueva Granada, Bogotá.

Barros, M. A. y Rojas, N. (2015). El rol de la mujer en el conflicto armado colombiano (tesis de maestría). Universidad Externado de Colombia, Bogotá.

Blake, D. D., Weathers, F. W., Nagy, L. M., Kaloupek, D. G., Gusman, F. D., Charney, D. S. y Keane, T. M. (1990). The development of a clinicianadministered PTSD scale. Journal of traumatic stress, 8(1), 75-90. Recuperado de https://link.springer.com/article/10.1007/BF02 105408

Botello, N. A. (2000). El trabajo de las mujeres policías. El Cotidiano, 16(101), 74-83.

Cadavid, M. R. (2014). Mujer: blanco del conflicto armado en Colombia. Analecta política, 4(7), 301-318. Recuperado de https://revistas.upb.edu.co/index.php/analecta/a rticle/view/2784/2430

Castañeda, J. y Camargo, J. (2018). Salud mental en población víctima del conflicto armado en Colombia. I+D Revista de Investigaciones, 12(2), 19-25. doi: https://doi.org/10.33304/revinv.v12n2-2018002

Castillo, A. y Palma, E. (2016). Resiliencia en mujeres viudas por el conflicto armado vinculadas 
Funvida en Tumaco (tesis de maestría). Pontificia Universidad Javeriana, Bogotá.

Correa, M. L., Restrepo, S., Enríquez, É., Pertuz, M. C., Montoya, S. y Acevedo, M. I. (2015). Las personas mayores víctimas del conflicto armado. Misión Colombia Envejece: Cifras, retos y recomendaciones. Recuperado de https://www.repository.fedesarrollo.org.co/han dle/11445/2736.

Cortez, L. Y. (2018). Efectividad, de los programas de emprendimiento y generación de ingresos para la población víctima del desplazamiento forzado en el municipio de Arauca. Interfaces, 1(1), 22$45 . \quad$ Recuperado de http://www.unilibrecucuta.edu.co/ojs/index.php /ingenieria/article/view/220

Cudris, L. y Barrios, Á. (2018). Malestar psicológico en víctimas del conflicto armado. Revista CS, (26), 75-90.

doi: https://doi.org/10.18046/recs.i26.3292

Escobar-Córdoba, F., Folino, J. O. y Schmalbach, J. E. (2006). Alteraciones del sueño y estrés postraumático en mujeres víctimas de asalto sexual. Medunab, 9(1), 28-33. Recuperado de https://revistas.unab.edu.co/index.php/medunab /article/view/3164/2662

Gómez, C., Tamayo, N., Buitrago, G., Guarnizo, C. C., Garzón, N., Eslava, J.,... y Rincón, C. J. (2016). Violencia por conflicto armado y prevalencias de trastornos del afecto, ansiedad y problemas mentales en la población adulta colombiana. Revista Colombiana de Psiquiatría, 45, 147153.

doi:

https://doi.org/10.1016/j.rcp.2016.11.001

Guevara, C. R. y Barney, A. F. (2009). Desplazamiento forzado en Florida, Valle del Cauca. Mujeres, territorio y cultura. Revista Científica Guillermo de Ockham, 7(1), 57-68. doi: https://doi.org/10.21500/22563202.535

Hmieleski, K. M. y Carr, J. C. (2008). The relationship between entrepreneur psychological capital and new venture performance. Frontiers of Entrepreneurship Research, 4, 1-15

Jara, A. (2017). En Colombia, 4.2 millones de víctimas del conflicto armado son mujeres. Bogotá:
Unidad de Víctimas. Recuperado de https://www.unidadvictimas.gov.co/es/enfoque s-diferenciales/en-colombia-42-millones-devictimas-del-conflicto-armado-son-mujeres$\underline{\text { alan }}$

Lubell, N. (2005). Challenges in applying human rights law to armed conflict. International review of the Red Cross, 87(860), 737-754.

Mariño-Arévalo, A. y Valencia-Toro, M. (2015). Participación de la gran empresa en la política pública de atención a las víctimas del conflicto armado en Colombia. Cuadernos de Administración, 28(50), 159-185. Recuperado de

http://www.scielo.org.co/pdf/cadm/v28n50/v28 $\underline{\text { n50a08.pdf }}$

Moreno, M. A. y Díaz, M. E. (2016). Posturas en la atención psicosocial a víctimas del conflicto armado en Colombia. El Ágora USB, 16(1), 193-213. Recuperado de http://www.scielo.org.co/scielo.php?pid=S1657

80312016000100010\&script=sci_abstract\&tlng $=\mathrm{es}$

Muñoz, L. (2018). Propuesta de innovación social para la inserción económica y social De mujeres adolescentes cabeza de familia víctimas del conflicto armado En San Vicente del Caguán Colombia (tesis de maestría). Universidad Externado de Colombia, Bogotá.

Pérez, K. y Salas, W. (2019). Ser o no ser: subjetivación política de las víctimas del conflicto armado en Colombia. En Carmona, J., y Moreno, F. (Eds), Reconstrucción de subjetividades e identidades en contextos de guerra y posguerra. (pp. 148166). Manizales, Caldas: Editorial Universidad de Manizales.

Ramírez, Y. (2016). Memoria y derechos humanos en el marco del conflicto armado colombiano. Principia Iuris, 13(26), 75-99. Recuperado de http://revistas.ustatunja.edu.co/index.php/piuris /article/view/1137/1102

Reyes, L. A. D., \& Fattori, G. (2019). Microfinance as a Means for Women Empowerment in the Colombian Post Conflict Scenario: Transformational Development or a Tool for 
ESTRÉS POSTRAUMÁTICO Y APTITUDES DE EMPRENDIMIENTO Y RESILIENCIA EN MUJERES VÍCTIMAS DEL CONFLICTO ARMADO

Better Managing Poverty?. Peace Human Rights Governance, 3(1), 127-161

Riaño, E. J. (2006). De "emprendedor" a" pobrecito" reestructuración del rol comunitario de familias campesinas desplazadas asentadas en los Altos de Cazuca Bogotá. Umbral científico, (9), 126$140 . \quad$ Recuperado de http://www.redalyc.org/pdf/304/30400914.pdf

Rodríguez, V. P. y Salazar, V. P. (2015). El crecimiento empresarial una herramienta fundamental para la reducción de la pobreza. Clío América, 9(17), $77 \quad-\quad 84 . \quad$ doi: https://doi.org/10.21676/23897848.1483

Romero, K. y Contreras, E. (2015). Revisión teórica sobre el post-conflicto: Una oportunidad para empoderar a mujeres víctimas de desplazamiento. Cultura Educación y Sociedad, 6(1), 79-92. Recuperado de https://revistascientificas.cuc.edu.co/culturaedu cacionysociedad/article/view/757

Romero, O. M., Jaramillo, M. F., Aguirre, M. C. y Ruiz, J. A. (2017). Resiliencia y percepción de oportunidades de negocio de los estudiantes de la Universidad Técnica de Machala, Ecuador. Revista Espacios, 38(50). Recuperado de https://www.revistaespacios.com/a17v38n50/a 17v38n50p23.pdf

Santamaría, M. F. (2016). El Marketing Social y su incidencia en el Emprendimiento Comercial de las niñas, adolescentes y mujeres víctimas de violencia intrafamiliar y sexual a través del Foro de la Mujer de la Provincia Bolívar (tesis de pregrado). Universidad Técnica de Ambato, Ambato.

Sierra, J. R. (2019). Breve historia del conflicto armado en Colombia. Madrid, España: Los Libros de la Catarata.

Singh, M. K. (2019). Explosive Remnants of War: Sheer Violation Of Human Rights. Research Journal of Social Sciences, 10(6), 149-158

Sinisterra, M., Figueroa, F., Moreno, V., Robayo, M. y Sanguino, J. (2010). Prevalencia del trastorno de estrés postraumático en población en situación de desplazamiento en la localidad de Ciudad Bolívar Bogotá, Colombia 2007. Psychologia,
4(2), 83-97. Recuperado de http://www.scielo.org.co/scielo.php?script=sci abstract\&pid $=$ S1900-

23862010000200008\&lng=es\&nrm=iso\&tlng= en

Tabares, C. M. (2011). Reflexiones en torno al devenir sujeto político de las víctimas del conflicto armado. Estudios Políticos, (38), 13-37. Recuperado

de http://www.redalyc.org/articulo.oa?id=164290 66002

Tol, W. A., Stavrou, V., Greene, M. C., Mergenthaler, C., Garcia-Moreno, C. y Van-Ommeren, M. (2013). Mental health and psychosocial support interventions for survivors of sexual and gender-based violence during armed conflict: a systematic review. World psychiatry: official journal of the World Psychiatric Association (WPA), 12(2), 179-180. doi: https://doi.org/10.1002/wps.20054

Uribe, M. M. (2017). El emprendimiento: algunas reflexiones desde un enfoque de revisión. Clío América, 11(22), 219-239. doi: https://doi.org/10.21676/23897848.2443

Utria, L., Amar, J., Martínez, M., Colmenares, G. y Crespo, F. (2015). Resiliencia en mujeres víctimas de desplazamiento forzado. Barranquilla, Colombia: Editorial Universidad del Norte.

Valencia, O. y Daza, M. (2010). Vinculación a grupos armados: un resultado del conflicto armado en Colombia. Diversitas: Perspectivas en Psicología, 6(2), 429-439. Recuperado de http://www.redalyc.org/html/679/67915140015 $\underline{1}$

Villa, J. D. (2012). La acción y el enfoque psicosocial de la intervención en contextos so- ciales: Podemos pasar de la moda a la precisión teórica, epistemológica y metodológica. El Ágora USB, 12(2), 349-365. Recuperado de http://www.scielo.org.co/scielo.php?pid=S1657 $\overline{80312012000200005 \& \text { script }=\text { sci_abstract\&tlng }}$ =es

Villa, G. J. (2016). Intervenciones psicosociales en el marco de acciones de reparación a víctimas del 
Kethy Luz Pérez-Correa, Oscar Eduardo Rodríguez-Vega y Belia Elena Linero-Gómez

conflicto armado colombiano. Estudios Centroamericanos, 71(744), 81-104. Recuperado de https://dialnet.unirioja.es/servlet/articulo?codig $\underline{\mathrm{o}=5793225}$

Villa, G. J., Barrera, M. D., Arroyave, P. L. y Montoya, B. Y. (2017). Acción con daño: del asistencialismo a la construcción social de la víctima. Mirada a procesos de reparación e intervención psicosocial en Colombia. Universitas Psychologica, 16(3), 1-13. doi: https://doi.org/10.11144/Javeriana.upsy163.adac

Villa, G. J., Londoño, N., Gallego, M., Arango, L. y Rosso, P. M. (2016). Apoyo mutuo, liderazgo afectivo y experiencia clínica comunitaria. Acompañamiento psicosocial para la "rehabilitación" de víctimas del conflicto armado. El Ágora USB, 16(2), 427-452. doi: https://doi.org/10.21500/16578031.2454

Viloria, J., Daza, A. y Miranda, L.F. (2016). Perfil emprendedor de los graduados de administración de empresas de la Universidad del Magdalena 2010-2014. Contexto, 5, 161171. doi: https://doi.org/10.18634/ctxj.5v.0i.584

Weathers, F., Litz, B., Herman, D., Huska, J. y Keane, T. (Octubre 1993). The PTSD Checklist (PCL): Reliability, Validity, and Diagnostic Utility. Conferencia presentada en la Annual Convention of the International Society for Traumatic Stress Studies, San Antonio

Williams, N., Vorley, T. y Ketikidis, PH. (2013). Resiliencia económica y emprendimiento: un estudio de caso de la región de la ciudad de Thessaloniki. Economía local, 28(4), 399-415.

Yaffe, L. (2011). Conflicto armado en Colombia: análisis de las causas económicas, sociales e institucionales de la oposición violenta. Revista CS, (8), 187-208. doi: http://dx.doi.org/10.18046/recs.i8.1133 